\title{
Interference Mitigation via Joint Detection
}

\author{
Jungwon Lee, Dimitris Toumpakaris, and Wei Yu
}

\begin{abstract}
This paper addresses the design of optimal and near-optimal detectors in an interference channel with fading and with additive white Gaussian noise (AWGN), where the transmitters employ discrete modulation schemes as in practical communication scenarios. The conventional detectors typically either ignore the interference or successively detect and then cancel the interference, assuming that the desired signal and/or the interference are Gaussian. This paper quantifies the significant performance gain that can be obtained if the detectors explicitly take into account the modulation formats of the desired and the interference signals. This paper first describes the optimal maximumlikelihood (ML) detector that minimizes the probability of detection error for a given modulation scheme, and the joint minimum-distance (MD) detector, which is a lower-complexity approximation of the ML detector. It is then demonstrated by analysis and by simulation that in an AWGN channel, while interference-ignorant and successive interference cancellation detectors are both prone to error floors, the optimal ML and joint MD detectors are not. This paper further analyzes the performance of joint detection in a Rayleigh fading environment. It is demonstrated that the joint detector can achieve symbol error rates that have the same dependence on the received signal-to-noise ratio (SNR) as if the channel were interference free. Thus, the performance of joint detection is fundamentally limited by the SNR rather than the signal-to-interference ratio (SIR). Moreover, the joint detector enables the use of transmit diversity schemes to achieve the same diversity order as in the absence of interference. These results show that the use of interference-aware detectors can significantly alleviate the effect of interference thereby improving the achievable rates and the reliability of future wireless systems.
\end{abstract}

\section{Index Terms}

interference channel, maximum likelihood detection, joint detection, inter-cell interference, crosstalk.

J. Lee is with Mobile Solutions Lab, Samsung Electornics US R\&D Center, 4921 Directors Pl, San Diego, CA 92121, USA e-mail: jungwon@stanfordalumni.org. D. Toumpakaris is with the Department of Electrical and Computer Engineering, University of Patras, Rio, Greece 26500 e-mail: dtouba@upatras.gr. W. Yu is with the Department of Electrical and Computer Engineering, University of Toronto, Toronto, Ontario, M5S 3G4, Canada e-mail: weiyu@ @comm.utoronto.ca. This paper has been presented in part at IEEE Int. Conf. Commun. (ICC), 2010.

Correspondence should be addressed to Jungwon Lee (jungwon@stanfordalumni.org). 


\section{INTRODUCTION}

The exponential growth of terrestrial wireless systems is expected to continue well into the coming decades. Current research and development efforts in future wireless systems have focused on achievable peak bit rates of up to $1 \mathrm{Gbit} / \mathrm{s}$ [1], [2], [3]. It is envisaged that the attainment of these rates will be facilitated by the deployment of distributed broadband wireless communications (BWC) systems [4], [5], where antennas acting as simple transmit/receive terminals are placed densely inside the coverage areas, and their signals are conveyed to central units with high-bandwidth links such as fiber, where they are processed jointly.

In order to take full advantage of the potential of a distributed antenna system, full (or at least, aggressive) frequency reuse is desirable. This is in contrast to the traditional practice in wireless system design, where interference is avoided by employing orthogonal multiplexing schemes (such as FDMA/TDMA/ CDMA/OFDMA) that do not achieve the highest possible area-spectral efficiency. For this reason, the design of wireless transceivers that have the ability to communicate reliably in the presence of interferers is of crucial importance in future wireless systems.

In conventional wireless transceiver design, interference is commonly viewed as contributing to an error floor at high signal-to-noise ratios (SNRs). However, this is true only if the detectors that are employed do not take into account the structure of the interference properly. In fact, in practical systems, interference may often be less detrimental than noise of equal power, because contrary to Gaussian noise, the signals emitted by the interferers belong to discrete constellations. The main point of this paper is to show that in many cases, the performance of the system is fundamentally limited by the SNR, rather than the signalto-interference ratio (SIR). For example, in a Rayleigh fading environment, the symbol error rate (SER) of an interference-aware detector can be made to decrease monotonically with the SNR, irrespective of the SIR.

A conventional detector typically uses one of the two following approaches when interference is present. When the SIR is relatively large, interference is treated as noise. This is the case, for example, in the development of power allocation algorithms for frequency-selective Gaussian interference channels (ICs) [6], [7], [8] and in early CDMA system design [9], [10]. When the SIR is small, successive interference cancellation (SIC) is applied: i.e. the interference is detected first then cancelled. However, in the AWGN channel, for some range of the SIR, both schemes suffer from an error floor. Thus, conventional detectors 
are typically interference-limited rather than noise-limited. The main result of this paper is that an interference-limited transmission system can be turned into a noise-limited one if a maximum-likelihood (ML) detector that incorporates knowledge of the interfering channel and the discrete nature of the interference is employed. Moreover, the ML detector can be approximated by a joint minimum-distance (MD) detector whose performance is almost the same for most SNR and SIR values.

The idea of joint detection has been extensively studied in the past both in the code-division multiple access (CDMA) context (e.g. [11], [12]) and in the single-user or multi-user multiple-input multipleoutput (MIMO) literature (e.g. [13], [14]), where the detector is typically interested in decoding several independently transmitted signals simultaneously. The setup for the IC differs in that only one of the transmitted signals is of interest. Thus, one might expect that treating interference as noise is optimal when the interference is low, while SIC is optimal when the interference is high. This paper shows that there exists an intermediate range of interference levels where neither ignoring nor completely decoding the interference is optimal. A good strategy in this regime is to detect the desired and the interfering signals jointly, then to discard the detector output for the interference (which may be unreliable anyway, but nevertheless aids the detection of the desired signal).

The existing literature on joint detection in the presence of inter-cell interference has typically focused on phase shift keying (PSK) modulation or performance measures other than the SER. For example, [15] examines the bit-error rate of ML and joint MD receivers for PSK modulation with the use of trellis coded modulation (TCM). In [16], the outage probability of multiuser detection is examined for PSK modulation with diversity. In [17], the spectral efficiency of multiuser detectors is computed by treating inter-cell interference as signals that need to be detected correctly (thus rendering an IC to a multipleaccess channel (MAC)). This paper deals with the average SER and SER upper bound in an IC with higher-order QAM modulation transmission, with possibly unequal power among the different users, and possibly with transmit diversity. To the best of the authors' knowledge, the performance analysis in such a setting has not appeared in the literature.

The analysis in this paper assumes uncoded signal constellation at the transmitter and hard decision decoding at the receiver. Soft decoding, which calculates a log-likelihood ratio (LLR) for each bit as the input to the decoder, would result in better performance than hard decoding, but its analysis also depends on the specific coding scheme. For this reason, this paper focuses on the analysis of hard decoding, which 
already provides insights on the receiver performance.

The optimal ML and the joint MD detectors are more complex to implement than the conventional detectors, but may not be significantly so when compared to the complexity of other parts of the receiver (such as a Viterbi decoder that may follow the detector). This is especially the case in a typical interference environment where only one or two interferers dominate. The significant performance improvement of joint detection may therefore justify its practical use. The optimal ML and the joint MD detectors also require synchronization, the knowledge of the modulation format of the interferers, and the knowledge of the interfering channel gains. Synchronization is a challenging issue, but is possible to implement in a distributed BWC system. The modulation information is usually heavily encoded in a preamble, so it can be decodable even at the non-intended receiver. Channel estimation in distributed BWC systems is typically made possible by appropriate and possibly adaptive placement of pilots (e.g., with fractional frequency reuse of pilots).

The remainder of this paper is organized as follows. The system model is presented in Section II. The conventional and the joint interference-aware detectors are described in detail in Sections III and IV, respectively. The main result of this paper, which is the performance analysis of the detectors, is presented in Sections V and VI for the AWGN and fading cases, respectively. The performance of the joint detector with transmit diversity is analyzed in Section VII. Simulation results are given in Section VIII. Finally, Section IX provides concluding remarks.

\section{SySTEM MODEL}

This paper considers the detection problem of the signal of user 1 in a $U$-user Gaussian IC. The received signal, $y[m]$, at time $m$ at receiver 1 is given by

$$
y[m]=\sum_{u=1}^{U} h_{u}[m] x_{u}[m]+z[m]
$$

where $x_{u}[m]$ is the signal of transmitter $u$ at time $m, h_{u}[m]$ is the gain of the channel from transmitter $u$ to receiver 1 , and $z[m]$ is the Gaussian background noise of receiver 1 with variance $N_{0} / 2$ per dimension. In vector form, (1) can be written as

$$
y=\left[\begin{array}{lll}
h_{1} & \cdots & h_{U}
\end{array}\right]\left[\begin{array}{lll}
x_{1} & \cdots & x_{U}
\end{array}\right]^{T}+z=\mathbf{h}^{*} \mathbf{x}+z,
$$

where $\mathbf{h}=\left[\begin{array}{lll}h_{1}^{*} & \cdots & h_{U}^{*}\end{array}\right]^{T}$ and $\mathbf{x}=\left[\begin{array}{lll}x_{1} & \cdots & x_{U}\end{array}\right]^{T}$. The transmit signal $x_{u}[m]$ is a symbol from a discrete signal constellation such as pulse-amplitude modulation (PAM), quadrature-amplitude modulation (QAM), 
and PSK. The power of the transmit signal is defined as $P_{u}=\mathbb{E}\left[\left|x_{u}\right|^{2}\right]$. In a Rayleigh fading channel, $h_{u} \sim \mathcal{C N}(0,1)$. For convenience, the following quantities are defined

$$
\mathrm{SNR} \triangleq \frac{P_{1}}{N_{0}}, \quad \mathrm{SIR} \triangleq \frac{P_{1}}{\sum_{u=2}^{U} P_{u}}, \quad \mathrm{SINR} \triangleq \frac{P_{1}}{\sum_{u=2}^{U} P_{u}+N_{0}}, \quad \text { and } \quad \mathrm{INR}_{u} \triangleq \frac{P_{u}}{N_{0}} .
$$

The problem of interest is the symbol-by-symbol detection of the transmit signal $x_{1}[m]$ using $y[m]$ at receiver 1 . It is assumed that receiver 1 knows the channel gains, $h_{u}[m]$, from all interferers and their modulation formats. These are typical assumptions for any advanced receiver for the IC [18]. This channel model is applicable to various practical systems, including distributed ones, for example, the downlink of a distributed BWC, where a mobile station needs to decode its own signal in the presence of interfering signals intended for other mobile stations.

\section{Conventional Detectors}

\section{A. Interference-Ignorant (II) Detector}

An interference-ignorant detector for $x_{1}$ simply divides the received signal $y$ by the direct channel gain $h_{1}$ then maps it to the closest point of the signal constellation of transmitter 1 . In other words, the interference term $\sum_{u=2}^{U} h_{u} x_{u}$ in (1) is lumped together with the background noise $z$.

In many realistic cellular and distributed BWC systems, there are typically only a few non-negligible interferers, each employing a discrete and finite constellation. In these cases, the receiver performance can be significantly improved by using interference-aware detectors that explicitly consider the discrete constellation. The SIC detector described in the following is such an interference-aware detector.

\section{B. SIC Detector}

Consider a two-user IC. Although the detector for receiver 1 is ultimately interested only in $x_{1}$, it can first obtain an estimate, $\hat{x}_{2}$, of $x_{2}$ by treating $x_{1}$ as noise, then detect $x_{1}$ based on $y-h_{2} \hat{x}_{2}$ by mapping $y-h_{2} \hat{x}_{2}$ to the closest constellation point scaled by $h_{1}$. Note that the SIC detector does not exploit the knowledge of the modulation scheme of $x_{1}$ when detecting $x_{2}$. Thus, the performance of SIC can be further improved if the discrete nature of both $x_{1}$ and $x_{2}$ is taken into account. 


\section{JOINT INTERFERENCE-AWARE DETECTORS}

\section{A. Optimal ML Detector}

The optimal maximum a posteriori (MAP) detector minimizes the probability of error $\operatorname{Pr}\left\{\hat{x}_{1} \neq x_{1}\right\}$ for the desired signal $x_{1}$, without the Gaussian assumption on either the desired signal or the interference. The probability of error can be expressed as

$$
P_{e} \triangleq \operatorname{Pr}\left\{\hat{x}_{1}(y) \neq x_{1}\right\}=\sum_{m_{1}=0}^{M_{1}-1} \operatorname{Pr}\left\{x_{1}=x_{1, m_{1}}\right\} \operatorname{Pr}\left\{\hat{x}_{1}(y) \neq x_{1, m_{1}} \mid x_{1}=x_{1, m_{1}}\right\},
$$

where $M_{1}$ is the constellation size of user 1 . When the constellation points of $x_{1}$ are equiprobable, the MAP detector reduces to the maximum-likelihood (ML) detector. By definition, the ML estimate of $x_{1}$ is

$$
\begin{gathered}
\hat{x}_{1}(y)=\underset{x_{1}}{\arg \max } f_{Y \mid X_{1}}\left(y \mid x_{1}\right), \text { where } \\
f_{Y \mid X_{1}}\left(y \mid x_{1}\right)=\sum_{m_{2}=0}^{M_{2}-1} \cdots \sum_{m_{U}=0}^{M_{U}-1} \operatorname{Pr}\left\{X_{2}=x_{2, m_{2}}, \cdots X_{U}=x_{U, m_{U}}\right\} \cdot f_{Y \mid X_{1}, \cdots, X_{U}}\left(y \mid x_{1}, \cdots, x_{U}\right) \\
=\frac{1}{M_{2} \cdots M_{U}} \sum_{m_{2}=0}^{M_{2}-1} \cdots \sum_{m_{U}=0}^{M_{U}-1} f_{Z}\left(y-h_{1} x_{1}-\sum_{u=2}^{U} h_{u} x_{u, m_{u}}\right) .
\end{gathered}
$$

Thus, the ML rule is

$$
\hat{x}_{1}(y)=\underset{x_{1}}{\arg \max } \sum_{m_{2}=0}^{M_{2}-1} \cdots \sum_{m_{U}=0}^{M_{U}-1} \exp \left(-\frac{\left|y-h_{1} x_{1}-\sum_{u=2}^{U} h_{u} x_{u, m_{u}}\right|^{2}}{N_{0}}\right)
$$

Note that the discrete constellations of all users are explicitly taken into account.

\section{B. Joint Minimum-Distance (MD) Detector}

The optimal ML detector requires the calculation of the sum of exponential functions and the calculation of the Euclidean distance from the received signal to all combined signal constellation points. In the following, a low-complexity detector is derived by simplifying the optimal ML detector.

When the noise power per dimension $N_{0} / 2$ is small, the following approximation can be used

$$
\sum_{m_{2}=0}^{M_{2}-1} \cdots \sum_{m_{U}=0}^{M_{U}-1} \exp \left(-\frac{\left|y-h_{1} x_{1}-\sum_{u=2}^{U} h_{u} x_{u, m_{u}}\right|^{2}}{N_{0}}\right) \approx \max _{x_{2}, \cdots, x_{U}} \exp \left(-\frac{\left|y-h_{1} x_{1}-\sum_{u=2}^{U} h_{u} x_{u, m_{u}}\right|^{2}}{N_{0}}\right) .
$$

Then the approximate ML detector can be expressed as

$$
\hat{x}_{1}(y)=\underset{x_{1}}{\arg \min }\left[\min _{x_{2}, \cdots, x_{U}}\left|y-h_{1} x_{1}-\sum_{u=2}^{U} h_{u} x_{u, m_{u}}\right|^{2}\right] .
$$


This approximate ML detector can be aptly named as joint MD detector because the estimate of $x_{1}$ is obtained from the combined signal constellation point of all users that is closest to the received signal.

The joint MD detector is similar to the optimal ML detector for the MAC where the receiver needs to minimize the decoding error of all received signals. However, in the IC, each receiver is interested only in decoding one signal, so the joint MD detector is not quite ML. The joint MD detector is easier to implement than the ML, and the difference in performance is small especially at large SNR. Further, unlike the SIC detector, the estimation of the desired signal in the joint MD detector does not rely on the correct estimate of the interfering signal. Hence, with the joint MD detector, the estimate of the desired signal may be correct even if the estimates of interfering signals are erroneous. Thus, unlike the sequential SIC, the joint MD detector does not suffer from error propagation.

\section{Performance Analysis of the Detectors in the AWGn Channel}

The joint MD and the optimal ML detectors are now compared analytically with the conventional detectors in terms of the SER. This section presents results for the AWGN case, followed by the fading case in the next section. For simplicity, only the 2-user IC is considered. This results in intuitive expressions. It is possible to extend the analysis to an IC with more than two users, although the expressions will be more complicated. The analysis for the 2-user IC is relevant when one single interferer dominates in the received signal. If more interferers are present, by adding their signals to the background noise, the 2-user analysis still gives a lower bound on the performance.

The key result here is that the performance of joint detectors is noise-limited, rather than interferencelimited. In an AWGN channel, joint detectors almost never exhibit an error floor. In a fading channel, the SER achieved by joint detectors is a function of the SNR rather than the SIR.

\section{A. SER with 2-PAM Used by Both Transmitters}

When 2-PAM is used by both the desired and the interfering transmitters, the SERs of the conventional and joint detectors can be easily derived using nearest neighbor union bound (NNUB) analysis. The expressions serve to highlight the difference among the detectors.

The SER of the interference-ignorant detector is

$$
P_{e, I I} \approx \begin{cases}\frac{1}{2} Q(\sqrt{\mathrm{SNR}}-\sqrt{\mathrm{INR}}), & \mathrm{SIR} \geq 1 \\ \frac{1}{2}-\frac{1}{2} Q(\sqrt{\mathrm{INR}}-\sqrt{\mathrm{SNR}}), & \mathrm{SIR}<1\end{cases}
$$


where $Q(x)=\frac{1}{\sqrt{2 \pi}} \int_{x}^{\infty} e^{-t^{2} / 2} d t$. The SER of the SIC detector is

$$
P_{e, S I C} \approx \begin{cases}\frac{1}{2} Q(\sqrt{\mathrm{SNR}}-2 \sqrt{\mathrm{INR}}), & \mathrm{SIR} \geq 4 \\ \frac{1}{2}-\frac{1}{2} Q(\sqrt{\mathrm{SNR}}-\sqrt{\mathrm{INR}}), & \frac{9}{4} \leq \mathrm{SIR}<4 \\ \frac{1}{2}-\frac{1}{2} Q(2 \sqrt{\mathrm{INR}}-\sqrt{\mathrm{SNR}}), & 1 \leq \mathrm{SIR}<\frac{9}{4} \\ \frac{1}{2} Q(\sqrt{\mathrm{INR}}-\sqrt{\mathrm{SNR}}), & \frac{1}{4} \leq \mathrm{SIR}<1 \\ Q(\sqrt{\mathrm{SNR}}), & \mathrm{SIR}<\frac{1}{4} .\end{cases}
$$

Lastly, the SER of the joint MD detector is given by

$$
P_{e, J M D} \approx \begin{cases}\frac{1}{2} Q(\sqrt{\mathrm{SNR}}-\sqrt{\mathrm{INR}}), & \mathrm{SIR} \geq 1 \\ \frac{1}{2} Q(\sqrt{\mathrm{INR}}-\sqrt{\mathrm{SNR}}), & \frac{1}{4} \leq \mathrm{SIR}<1 \\ Q(\sqrt{\mathrm{SNR}}), & \mathrm{SIR}<\frac{1}{4} .\end{cases}
$$

The SER of the optimal ML detector is harder to derive, but is upper bounded by the SER of the joint MD detector: $P_{e, M L} \leq P_{e, J M D}$.

It is interesting to examine the behavior of the near-optimal joint MD detector for various SIR levels. From (12), it is clear that, for $\operatorname{SIR} \geq 1$, the joint MD detector behaves as if the minimum distance of the constellation of the desired signal is reduced by the interference. For $\frac{1}{4} \leq \operatorname{SIR}<1$, it behaves as if the minimum distance of the constellation of the interference is reduced by the desired signal. For $\operatorname{SIR}<\frac{1}{4}$, the detection of the desired signal is not affected by the (very strong) interference.

Comparing (10) and (12), it can be seen that the joint MD detector outperforms the conventional interference-ignorant detector for SIR $<1$. Comparing (11) and (12), it is clear that the joint MD detector outperforms the SIC detector for SIR $\geq 1$. The performance of the SIC detector is particularly poor in the region $1 \leq \mathrm{SIR}<4$. However, it can also be seen that when both transmitters employ 2-PAM, joint detection is equivalent to the ordered SIC with optimal ordering based on whether SIR is greater than or smaller than 1. This, however, is not the case when higher-order modulation is used.

\section{B. Error Floor Analysis with Higher-Order Modulation}

Unlike the 2-PAM case, the joint MD detector can outperform the ordered SIC detector significantly when higher-order modulation is employed. This is explained here with an example. Assume that 2-PAM is used for the desired signal, but 4-PAM is used for the interference, i.e., $x_{1}= \pm 1$ and $x_{2}= \pm \frac{1}{\sqrt{5}}$ or $\pm \frac{3}{\sqrt{5}} \cdot h_{1}=4$ and $h_{2}=3 \sqrt{5}$ and the background noise is assumed to be zero. When $\left(x_{1}, x_{2}\right)=\left(-1, \frac{3}{\sqrt{5}}\right)$, 
$y=h_{1} x_{1}+h_{2} x_{2}=5$. The interference-ignorant detector would declare $\hat{x}_{1}=1\left(\neq x_{1}\right)$ because $y>0$. In the case of the SIC detector, the estimate of the interference is $\hat{x}_{2}=\frac{1}{\sqrt{5}}\left(\neq x_{2}\right)$ because the decision boundaries for the interference are located at $y=0$ and $y= \pm 3 \sqrt{5} \cdot \frac{1}{2}\left(\frac{1}{\sqrt{5}}+\frac{3}{\sqrt{5}}\right)= \pm 6$, and $0 \leq y=5<6$. Then after interference subtraction $\tilde{y}=y-h_{2} \hat{x}_{2}=5-3 \sqrt{5} \cdot \frac{1}{\sqrt{5}}=2>0$. Since $\tilde{y}>0, \hat{x}_{1}=1\left(\neq x_{1}\right)$. Thus, both conventional detectors fail. But, it is clear that the joint MD detector and the optimal ML detector estimate the transmit signal correctly in this zero-noise case.

In general, when a signal constellation larger than 2-PAM is used, determining the optimal ordering for SIC is not trivial. Moreover, even given the optimal ordering, the ordered SIC detector does not perform as well as the optimal ML (or the joint MD) detector. To quantify the performance advantage of the ML and joint MD detectors over the conventional ones, the following result provides an asymptotic error-floor analysis for any arbitrary PAM constellation. This asymptotically high SNR case is of practical interest for distributed BWC systems with densely placed antennas, where interference is much larger than noise.

Proposition 1. Consider the 2-user Gaussian IC where the desired and the interfering user employ $M_{1^{-}}$ and $M_{2}$-PAM, respectively. For the interference-ignorant (II) detector, there exists a threshold $\mathrm{SIR}_{t h, I I}$, given by

$$
\operatorname{SIR}_{t h, I I}=\left(M_{1}+1\right)\left(M_{1}-1\right)\left(M_{2}-1\right) /\left(M_{2}+1\right),
$$

such that, when $\operatorname{SIR} \leq \mathrm{SIR}_{t h, I I}$, the SER reaches a non-zero error floor as SNR increases to infinity. For the SIC detector, there exist threshold values $\operatorname{SIR}_{t h, S I C, 1}$ and $\mathrm{SIR}_{t h, S I C, 2}$ given by

$$
\begin{aligned}
& \operatorname{SIR}_{t h, S I C, 1}=\left(M_{1}+1\right) /\left\{\left(M_{1}-1\right)\left(M_{2}-1\right)\left(M_{2}+1\right)\right\}, \text { and } \\
& \operatorname{SIR}_{t h, S I C, 2}=4\left(M_{1}+1\right)\left(M_{1}-1\right)\left(M_{2}-1\right) /\left(M_{2}+1\right),
\end{aligned}
$$

such that, when $\mathrm{SIR}_{t h, S I C, 1} \leq \mathrm{SIR} \leq \mathrm{SIR}_{t h, S I C, 2}$, the SER reaches a non-zero error floor as $\mathrm{SNR} \rightarrow \infty$. The optimal ML and joint MD detectors do not exhibit error floors, except for a finite number of SIR values at which the received constellation points for different values of the desired signals are collocated.

Proof: Consider the ML and the joint MD detector first. There are $M_{1} \cdot M_{2}$ decision regions, one for each combined constellation symbol. Clearly, as SNR $\rightarrow \infty$, the perturbation of the combined received symbol caused by Gaussian noise becomes vanishingly small compared to the radius of the decision region. Hence, $P_{e, M D} \rightarrow 0$ regardless of the value of the SIR. Note that there is a finite set of SIR values for which the above do not hold. When the SIR is such that some of the combined points overlap, an error floor appears. For example, when both users employ 2-PAM and SIR $=0 \mathrm{~dB},\left(x_{1}=+1, x_{2}=-1\right)$ 
and $\left(x_{1}=-1, x_{2}=+1\right)$ are both mapped to the combined symbol 0 . Clearly, in these cases, an error floor appears because some input combinations cannot be distinguished, even in the absence of noise.

For the interference-ignorant detector, the region where an SER floor appears can be found by first noting that, in the absence of signals from other users and noise, the distance between two neighboring points of the PAM constellation of user 2 at receiver 1 is equal to $d_{2}$, where $d_{u}=\sqrt{12 h_{u}^{2} /\left(M_{u}^{2}-1\right)}$ [19]. Moreover, the distance of the outermost symbol of the PAM constellation of user 2 from the constellation center is equal to $r_{2}$, where $r_{u}=\frac{M_{u}-1}{2} d_{u}$. Then errors will occur when symbols sent by user 1 cross the boundary of their corresponding decision region. In the absence of noise, this can only happen when $r_{2}$ exceeds $d_{1} / 2$. Using the expressions for $r_{2}$ and $d_{2}, r_{2} \geq d_{1} / 2 \Rightarrow \frac{\left(M_{2}-1\right)}{2} \sqrt{\left(12 h_{2}^{2}\right) /\left(M_{2}^{2}-1\right)} \geq \frac{1}{2} \sqrt{\left(12 h_{1}^{2}\right) /\left(M_{1}^{2}-1\right)}$. By rearranging and noting that $\operatorname{SIR}=h_{1}^{2} / h_{2}^{2}$, (13) is obtained.

Finally, the error floor of a SIC detector is analyzed. For an error floor to occur, in the absence of noise, both conditions below should hold:

1) The received symbol $y=h_{1} x_{1}+h_{2} x_{2}$ is outside the decision region of $x_{2}$ that was actually transmitted, AND

2) After subtraction of $\hat{x}_{2}, y-h_{2} \hat{x}_{2}$ is outside the decision region of $x_{1}$ that was actually transmitted. Note that 2) cannot hold if 1) does not hold, because the noise is assumed to be zero.

In order for 1) to be true, $h_{1} x_{1}+h_{2} x_{2}>h_{2}\left(x_{2}+d_{2} / 2\right)$ or $h_{1} x_{1}+h_{2} x_{2}<h_{2}\left(x_{2}-d_{2} / 2\right)$, where $d_{2}$ is the minimum distance of the constellation of the interferer. Equivalently, $h_{1}^{2} x_{1}^{2}>h_{2}^{2} d_{2}^{2} / 4 \Rightarrow \mathrm{SIR}=\frac{h_{1}^{2}}{h_{2}^{2}} \geq \frac{d_{2}^{2}}{4 x_{1}^{2}}$. Using the largest possible value of $x_{1}^{2}=\left(\left(M_{1}-1\right) d_{1} / 2\right)^{2}$, which gives the lower bound,

$$
\mathrm{SIR} \geq \frac{1}{4} \frac{12}{\left(M_{2}^{2}-1\right)} \frac{4\left(M_{1}^{2}-1\right)}{12\left(M_{1}-1\right)^{2}}=\frac{M_{1}+1}{\left(M_{1}-1\right)\left(M_{2}+1\right)\left(M_{2}-1\right)} \triangleq \operatorname{SIR}_{t h, S I C, 1}
$$

In order for 2) to hold, $y-h_{2} \hat{x}_{2}=h_{1} x_{1}+h_{2}\left(x_{2}-\hat{x}_{2}\right) \leq h_{1}\left(x_{1}-d_{1} / 2\right)$ or $h_{1} x_{1}+h_{2}\left(x_{2}-\hat{x}_{2}\right) \geq h_{1}\left(x_{1}+\right.$ $\left.d_{1} / 2\right) \Rightarrow h_{2}^{2}\left(x_{2}-\hat{x}_{2}\right)^{2} \geq h_{1}^{2} d_{1}^{2} / 4 \Rightarrow \operatorname{SIR}=\frac{h_{1}^{2}}{h_{2}^{2}} \leq \frac{4\left(x_{2}-\hat{x}_{2}\right)^{2}}{d_{1}^{2}}$. For the higher bound, $x_{2}=-\hat{x}_{2}=x_{2, \max }$. Therefore,

$$
\mathrm{SIR} \leq 4\left(4 \cdot \frac{12\left(M_{2}-1\right)^{2}}{4\left(M_{2}^{2}-1\right)}\right) \frac{M_{1}^{2}-1}{12}=4 \frac{\left(M_{1}-1\right)\left(M_{1}+1\right)\left(M_{2}-1\right)}{M_{2}+1} \triangleq \operatorname{SIR}_{t h, S I C, 2},
$$

which completes the proof.

It can be easily shown that, for $M_{1} \geq 2$ and $M_{2} \geq 2$

$$
\mathrm{SIR}_{t h, S I C, 1} \leq \operatorname{SIR}_{t h, I I}<\operatorname{SIR}_{t h, S I C, 2}=4 \operatorname{SIR}_{t h, I I}
$$


where the equality between $\operatorname{SIR}_{t h, S I C, 1}$ and $\operatorname{SIR}_{t h, I I}$ holds only for $\left(M_{1}, M_{2}\right)=(2,2)$. Thus, both the interference-ignorant detector and the SIC detector have an error floor for $\mathrm{SIR}_{t h, S I C, 1} \leq \mathrm{SIR} \leq \mathrm{SIR}_{t h, I I}$. Table I shows some sample SIR threshold values. As can be seen in the table, the gap between SIR $_{t h, S I C, 1}$ and $\operatorname{SIR}_{t h, I I}$ increases as $\left(M_{1}, M_{2}\right)$ increases. Thus, for larger constellation sizes, the optimal ML or the joint MD detector should be employed instead of the conventional detectors.

The analysis of the $U$-user case is, in principle, a simple extension of the 2-user case, but the resulting expressions are more complicated. For example, with the interference-ignorant detector, there exists an error floor when $\sum_{u=2}^{U} r_{u} \geq \frac{d_{1}}{2}$, i.e., $\sum_{u=2}^{U} \sqrt{\frac{M_{u}-1}{M_{u}+1} \frac{1}{\operatorname{SIR}_{u}}} \geq \frac{1}{\sqrt{M_{1}^{2}-1}}$, where $\operatorname{SIR}_{u} \triangleq h_{1}^{2} / h_{u}^{2}$.

The aforementioned results shed light on the structure of optimal detection in the IC. On one hand, the fact that the joint detector outperforms the interference-ignorant detector implies that detecting interference is beneficial even when the interference is not of interest. On the other hand, the fact that the joint detector outperforms SIC suggests that successive cancellation is too aggressive in relying on the successful detection of the entire interference signal. (This was also recognized in [20] where a weighting factor is introduced to alleviate error propagation.) Instead, joint detection should be applied, which allows "soft" decoding of the interference signal to achieve the optimal performance.

\section{Performance Analysis in the Fading Interference Channel}

This section analyzes the performance of various detectors in the fading IC. The primary focus here is the interference-ignorant and the joint MD detector. The joint MD detector is considered instead of the ML and SIC detectors, because the joint MD detector has almost the same performance as the optimal ML detector except at very low SNR. Moreover, the joint MD detector significantly outperforms the SIC detector, which requires the same kind of channel and modulation information for the interfering users.

The main result of this section is that the SER of the joint MD detector in a fading IC can be approximated by an expression of the form $c /$ SNR, similar to that in a channel without interference. The penalty due to the presence of interference is accounted for by the coefficient $c$, which depends on the interference, but is upper-bounded by a finite value regardless of the value of the SIR.

\section{A. SER in the Absence of Interference}

It is useful to establish first the average SER for a fading channel in the absence of interference. In this case, the received signal is $y=h_{1} x_{1}+z$, where $h_{1} \sim \mathcal{C N}(0,1), z \sim \mathcal{C N}\left(0, N_{0}\right)$, and $\mathbb{E}\left[\left|x_{1}\right|\right]^{2}=P_{1}$. The 
average SER can be derived by considering the conditional SER given $h_{1}$ and then taking the average over the distribution of $h_{1}$ :

$$
\operatorname{Pr}\left\{\hat{x}_{1} \neq x_{1}\right\}=\mathbb{E}_{h_{1}}\left[\operatorname{Pr}\left\{\hat{x}_{1} \neq x_{1} \mid h_{1}\right\}\right]
$$

For a given $h_{1}$, and $M_{1}$-QAM, the conditional SER can be evaluated using the NNUB approach as follows:

$$
\operatorname{Pr}\left\{\hat{x}_{1} \neq x_{1} \mid h_{1}\right\} \leq 4\left(1-\frac{1}{\sqrt{M_{1}}}\right) Q\left(\sqrt{\frac{3\left|h_{1}\right|^{2} \mathrm{SNR}}{M_{1}-1}}\right),
$$

where $4\left(1-1 / \sqrt{M_{1}}\right)$ represents the average number of nearest neighbors over all constellation points. It is also well known that, when $h_{1} \sim \mathcal{C N}(0,1)$ [21],

$$
\begin{gathered}
\mathbb{E}_{h_{1}}\left[Q\left(\sqrt{a\left|h_{1}\right|^{2}}\right)\right]=\frac{1}{2} q(a), \text { where } \\
q(a)=1-\sqrt{\frac{a / 2}{1+a / 2}} .
\end{gathered}
$$

From (18), (19), and (20), the following upper bound can be obtained for the average SER

$$
p_{e, \text { no int,UB }}=2\left(1-\frac{1}{\sqrt{M_{1}}}\right) q\left(\frac{3 \mathrm{SNR}}{M_{1}-1}\right) .
$$

To gain more insight on the average SER, a simpler approximate expression is derived using the Taylor series expansion of $q(a)$ around $1 / a=0$. For large $a, q(a) \approx 1 / a$. Then an approximate upper bound is

$$
p_{e, \text { no int,AUB }}=2\left(1-\frac{1}{\sqrt{M_{1}}}\right) \frac{M_{1}-1}{3 \mathrm{SNR}} .
$$

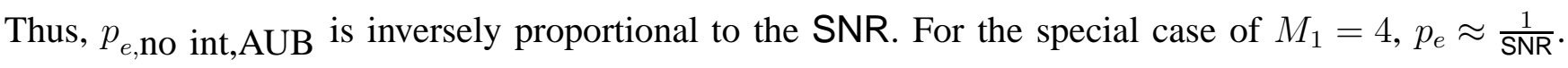

A lower bound of the average SER can be obtained by considering only half the nearest neighbors. Thus, the lower bound is exactly half the upper bound, and both have the form $c / \mathrm{SNR}$. This is true even in the presence of interference. Thus, in the remainder of this section, only upper bounds are examined.

\section{B. SER for the Interference-Ignorant (II) Detector}

The interference-ignorant detector with no knowledge on the channel gains $h_{2}, \cdots, h_{U}$ or the modulation formats of $x_{2}, \cdots, x_{U}$ ignores the presence of interference and makes a decision on $x_{1}$ based on the direct channel gain, $h_{1}$, and the modulation format of $x_{1}$ only. In general, the exact analysis of the interferenceignorant detector is cumbersome in the case of a fading channel. However, for the special case when all 
interferers employ PSK, the average SER performance can be easily analyzed. With $x_{u}$ a PSK symbol, $h_{u} x_{u}$ is a circularly symmetric complex Gaussian random variable: $h_{u} x_{u} \sim \mathcal{C N}\left(0, P_{u}\right)$. Thus

$$
y=h_{1} x_{1}+w,
$$

where $w \sim \mathcal{C N}\left(0, \sum_{u=2}^{U} P_{u}+N_{0}\right)$. Then the average SER, when $x_{1}$ uses $M_{1}$-QAM, is upper-bounded by

$$
p_{e, \mathrm{II}, \mathrm{UB}}=2\left(1-\frac{1}{\sqrt{M_{1}}}\right) q\left(\frac{3 \mathrm{SINR}}{M_{1}-1}\right),
$$

which, for large SINR, can be approximated by

$$
p_{e, \mathrm{II}, \mathrm{App} . \mathrm{UB}}=2\left(1-\frac{1}{\sqrt{M_{1}}}\right) \frac{M_{1}-1}{3 \mathrm{SINR}} .
$$

Therefore, the SER of the interference-ignorant detector is limited by the SINR instead of the SNR, unlike the case of no interference. In Section VIII, using 16-QAM as an example, it is shown by simulation that the dependence on 1/SINR is maintained when QAM is employed by the interferer instead of PSK.

\section{SER for the Joint MD (JMD) Detector}

An upper bound is now derived for the SER of the joint MD detector. However, the NNUB approach used earlier is complicated by the fact that the nearest neighbors change as the channel gains vary in the presence of interference. Moreover, the combined constellation $\sum_{u=1}^{U} h_{u} x_{u}$ of the received signal is no longer square-shaped even when the constellation of each individual $x_{u}$ is. Thus, counting the nearest neighbors does not yield a strict upper bound. To address this issue, this paper takes the approach of considering not only the nearest neighbors, but all signal constellation points that can possibly result in an error, even though the upper bound obtained in this way may not be the tightest.

With the above new approach in mind, first, the pairwise error probability (PEP) between two transmit symbol vectors is considered for a fixed $\mathbf{h}$. Then this PEP is averaged over $\mathbf{h}$, leading to a simple expression for the average PEP. Lastly, the upper bound of the average SER is calculated using the average PEP by considering all possible transmit symbol vectors.

The PEP for a given $\mathbf{h}$ is derived by examining the simple detection problem where the transmit vector $\mathrm{x}$ is either $\mathrm{x}_{A}$ or $\mathrm{x}_{B}$. The ML detector for this problem selects the transmit vector $\hat{\mathbf{x}}$ that results in $\mathbf{h}^{*} \hat{\mathbf{x}}$ closest to $y$. Hence the PEP, i.e., the probability that $\mathbf{x}_{B}$ is erroneously detected given that $\mathbf{x}_{A}$ was transmitted is

$$
P\left\{\mathbf{x}_{A} \rightarrow \mathbf{x}_{B} \mid \mathbf{h}\right\}=Q\left(\frac{\left\|\mathbf{h}^{*}\left(\mathbf{x}_{A}-\mathbf{x}_{B}\right)\right\|}{2 \sqrt{N_{0} / 2}}\right) .
$$


The average PEP over all channel realizations is

$$
\operatorname{Pr}\left\{\mathbf{x}_{A} \rightarrow \mathbf{x}_{B}\right\}=\mathbb{E}_{\mathbf{h}}\left[Q\left(\sqrt{\frac{\mathbf{h}^{*}\left(\mathbf{x}_{A}-\mathbf{x}_{B}\right)\left(\mathbf{x}_{A}-\mathbf{x}_{B}\right)^{*} \mathbf{h}}{2 N_{0}}}\right)\right] .
$$

The matrix $\left(\mathbf{x}_{A}-\mathbf{x}_{B}\right)\left(\mathbf{x}_{A}-\mathbf{x}_{B}\right)^{*}$ is Hermitian. So, it can be written as

$$
\left(\mathbf{x}_{A}-\mathbf{x}_{B}\right)\left(\mathbf{x}_{A}-\mathbf{x}_{B}\right)^{*}=\mathbf{U} \Lambda \mathbf{U}^{*}
$$

where $\mathbf{U}$ is a unitary matrix, i.e., $\mathbf{U}^{*} \mathbf{U}=\mathbf{U U}^{*}=\mathbf{I}$, and $\boldsymbol{\Lambda}$ is a diagonal matrix, i.e., $\boldsymbol{\Lambda}=\operatorname{diag}\left\{\lambda_{1}^{2}, \cdots, \lambda_{U}^{2}\right\}$. By defining $\tilde{\mathbf{h}}$ as $\mathbf{U}^{*} \mathbf{h}$, the average PEP can be represented as

$$
\operatorname{Pr}\left\{\mathbf{x}_{A} \rightarrow \mathbf{x}_{B}\right\}=\mathbb{E}_{\tilde{\mathbf{h}}}\left[Q\left(\sqrt{\frac{\tilde{\mathbf{h}}^{*} \boldsymbol{\Lambda} \tilde{\mathbf{h}}}{2 N_{0}}}\right)\right]=\mathbb{E}_{\tilde{\mathbf{h}}}\left[Q\left(\sqrt{\frac{\sum_{u=1}^{U}\left|\tilde{h}_{i}\right|^{2} \lambda_{i}^{2}}{2 N_{0}}}\right)\right],
$$

where $\tilde{\mathbf{h}}$ has the same distribution as $\mathbf{h}$ since $h_{u}$ for $u=1, \cdots, U$ are i.i.d. $\mathcal{C N}(0,1)$ and $\mathbf{U}$ is unitary.

Because $\left(\mathbf{x}_{A}-\mathbf{x}_{B}\right)\left(\mathbf{x}_{A}-\mathbf{x}_{B}\right)^{*}$ has rank $1, \lambda_{u}=0$ for $u=2, \cdots, U$. It can also be seen easily that $\lambda_{1}=\left\|\mathbf{x}_{A}-\mathbf{x}_{B}\right\|$ (the Euclidean distance between $\mathbf{x}_{A}$ and $\mathbf{x}_{B}$ ). Thus, as is well known from the analysis of Rayleigh fading channels [21], the average PEP can be expressed in closed form as

$$
\operatorname{Pr}\left\{\mathbf{x}_{A} \rightarrow \mathbf{x}_{B}\right\}=\frac{1}{2} q\left(\frac{\lambda_{1}^{2}}{2 N_{0}}\right)=\frac{1}{2} q\left(\frac{\left\|\mathbf{x}_{A}-\mathbf{x}_{B}\right\|^{2}}{2 N_{0}}\right) .
$$

Using this expression for the average PEP, it is now possible to derive an upper bound for the average SER for the detection of $x_{1}$. In the following, the upper bound is first derived for the 2-user case with both users employing 4-QAM. Then an upper bound is derived for any constellation sizes.

From the symmetry of 4-QAM, the average PEP does not depend on the transmitted symbol. Thus,

$$
\operatorname{Pr}\left\{\hat{x}_{1} \neq x_{1}\right\}=\operatorname{Pr}\left\{\hat{x}_{1} \neq x_{1,00} \mid \mathbf{x}=\mathbf{x}_{0000}\right\}
$$

where $\mathbf{x}_{p q r s}=\left[\begin{array}{ll}x_{1, p q} & x_{2, r s}\end{array}\right]^{T}$ and $x_{1, p q}$ and $x_{2, r s}$ are Gray-coded. In other words,

$$
x_{1, p q}=\left\{\begin{array}{ll}
\sqrt{\frac{P_{1}}{2}}(1+j), & \text { for }(p, q)=(0,0) \\
\sqrt{\frac{P_{1}}{2}}(-1+j), & \text { for }(p, q)=(0,1) \\
\sqrt{\frac{P_{1}}{2}}(-1-j), & \text { for }(p, q)=(1,1) \\
\sqrt{\frac{P_{1}}{2}}(1-j), & \text { for }(p, q)=(1,0)
\end{array} .\right.
$$

$x_{2, r s}$ can be similarly represented. Then

$$
\begin{aligned}
\operatorname{Pr}\left\{\hat{x}_{1} \neq x_{1,00} \mid \mathbf{x}=\mathbf{x}_{0000}\right\} \leq & \sum_{a=0}^{1} \sum_{b=0}^{1}\left[P\left\{\mathbf{x}_{0000} \rightarrow \mathbf{x}_{10 a b}\right\}+P\left\{\mathbf{x}_{0000} \rightarrow \mathbf{x}_{01 a b}\right\}\right] \\
& +P\left\{\mathbf{x}_{0000} \rightarrow \mathbf{x}_{1101}\right\}+P\left\{\mathbf{x}_{0000} \rightarrow \mathbf{x}_{1110}\right\}+P\left\{\mathbf{x}_{0000} \rightarrow \mathbf{x}_{1111}\right\}
\end{aligned}
$$


where $\operatorname{Pr}\left\{\mathbf{x}_{0000} \rightarrow \mathbf{x}_{1100}\right\}$ was not included because the error event of $\mathbf{x}_{0000} \rightarrow \mathbf{x}_{1100}$ is covered by the error events of $\mathrm{x}_{0000} \rightarrow \mathrm{x}_{1000}$ and $\mathrm{x}_{0000} \rightarrow \mathrm{x}_{0100}$. Each term in the upper bound (34) can be evaluated using the average PEP (31). The squared Euclidean distance between $\mathbf{x}_{p q r s}$ and $\mathbf{x}_{0000}$ is

$$
\left\|\mathbf{x}_{p q r s}-\mathbf{x}_{0000}\right\|^{2}=d_{1, p q}^{2}+d_{2, r s}^{2}
$$

where $d_{u, 00}^{2}=0, d_{u, 01}^{2}=d_{u, 10}^{2}=2 P_{u}$, and $d_{u, 11}^{2}=4 P_{u}$ for $u=1,2$. Thus, the upper bound of the average SER of the joint MD detector is

$$
p_{e, 1, \mathrm{JMD}, \mathrm{UB}}=q(\mathrm{SNR})+2 q(\mathrm{SNR}+\mathbf{I N R})+q(\mathrm{SNR}+2 \mathrm{INR})+q(2 \mathrm{SNR}+\mathbf{I N R})+\frac{1}{2} q(2 \mathrm{SNR}+2 \mathrm{INR}) .
$$

For sufficiently high SNR, the upper bound can be approximated as follows:

$$
p_{e, 1, \mathrm{JMD}, \mathrm{UB}} \approx \frac{1}{\mathrm{SNR}}+\frac{2}{\mathrm{SNR}+\mathrm{INR}}+\frac{1}{\mathrm{SNR}+2 \mathrm{INR}}+\frac{1}{2 \mathrm{SNR}+\mathrm{INR}}+\frac{1}{4 \mathrm{SNR}+4 \mathrm{INR}}=\frac{f(\mathrm{SIR})}{\mathrm{SNR}},
$$

where

$$
f(\alpha)=1+\frac{\alpha}{2 \alpha+1}+\frac{9}{4} \cdot \frac{\alpha}{\alpha+1}+\frac{\alpha}{\alpha+2} .
$$

Hence, at sufficiently high SNR, for fixed SIR, $p_{e, 1, \mathrm{JMD}}$,UB is inversely proportional to SNR. The coefficient $f(\alpha)$ in (37) is monotonically increasing in $\alpha$ for $\alpha \geq 0$, i.e., $f^{\prime}(\alpha)>0$ for $\alpha \geq 0$. Moreover,

$$
f(0)=1 \leq f(\alpha)<\frac{19}{4}=f(\infty) .
$$

Hence, regardless of the value of SIR,

$$
\operatorname{Pr}\left\{\hat{x}_{1} \neq x_{1}\right\} \lesssim \frac{19}{4 \mathrm{SNR}} .
$$

Note that $f(\infty)=19 / 4$ is an overestimate, because all the possible pairwise error events (and not just nearest neighbors) are considered for the derivation of the upper bound. When $\operatorname{SIR}=\infty$, the actual SER should be $\operatorname{Pr}\left\{\hat{x}_{1} \neq x_{1}\right\} \approx \frac{1}{\mathrm{SNR}}$.

The above analysis sheds light on the behavior of the average SER when user 1 and user 2 employ a constellation of size $M_{1}$ and $M_{2}$, respectively, in which case $\left(M_{1}-1\right) M_{2}$ PEP terms need to be considered for the calculation of the average SER upper bound. Note that, for any $\mathbf{x}_{A}$ and $\mathbf{x}_{B}$,

$$
\left\|\mathbf{x}_{A}-\mathbf{x}_{B}\right\| \geq\left|x_{1, A}-x_{1, B}\right|,
$$

where $x_{1, A}$ and $x_{1, B}$ are the symbols of user 1 corresponding to $\mathbf{x}_{A}$ and $\mathbf{x}_{B}$, respectively. Furthermore,

$$
\left|x_{1, A}-x_{1, B}\right| \geq \sqrt{\frac{6 P_{1}}{M_{1}-1}},
$$


where $\frac{6 P_{1}}{M_{1}-1}$ is the minimum distance between any pair of signal constellation points of user 1 . Then the average PEP bound becomes

$$
\operatorname{Pr}\left\{\mathbf{x}_{A} \rightarrow \mathbf{x}_{B}\right\}=\frac{1}{2} q\left(\frac{\left\|\mathbf{x}_{A}-\mathbf{x}_{B}\right\|^{2}}{2 N_{0}}\right) \leq \frac{1}{2} q\left(\frac{3 \mathrm{SNR}}{M_{1}-1}\right),
$$

since $q(x)$ is a monotonically decreasing function of $x$. Thus,

$$
p_{e, 1, \mathrm{JMD}, \mathrm{UB}}=\frac{\left(M_{1}-1\right) M_{2}}{2} q\left(\frac{3 \mathrm{SNR}}{M_{1}-1}\right) \approx \frac{\left(M_{1}-1\right)^{2} M_{2}}{6 \mathrm{SNR}},
$$

for sufficiently large SNR. In the special case where $\left(M_{1}, M_{2}\right)=(4,4)$, the above bound becomes

$$
p_{e, 1, \mathrm{JMD}, \mathrm{UB}}=6 q(\mathrm{SNR}) \approx \frac{6}{\mathrm{SNR}} .
$$

This upper bound is looser than the bound of (40) because the contribution of $\left|x_{2, A}-x_{2, B}\right|$ was ignored in (41) and the minimum distance was used instead of the actual distance $\left|x_{1, A}-x_{1, B}\right|$ in (42). If a tighter bound is desired, the actual distance $\left|x_{1, A}-x_{1, B}\right|$ can be calculated in a straightforward way. For example, for $M_{1}=16$, the following tighter approximate upper bound can be obtained:

$$
p_{e, 1, \mathrm{JMD}, \mathrm{UB}, 16-\mathrm{QAM}} \approx \frac{2965 M_{2}}{208 \mathrm{SNR}} \approx \frac{14.3 M_{2}}{\mathrm{SNR}},
$$

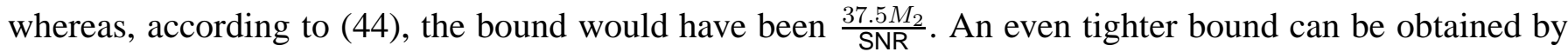
considering $\left|x_{2, A}-x_{2, B}\right|$. Nonetheless, the bound (44) shows that the average SER decreases according to $c /$ SNR, where $c$ depends on the constellation sizes of the signal of interest and the interferer.

The average SER bound (44) can be compared with (22) in the absence of interference:

$$
\frac{p_{e, 1, \mathrm{JMD}, \mathrm{UB}}}{p_{e, \mathrm{no} \text { int, } \mathrm{UB}}}=\frac{\sqrt{M_{1}}\left(\sqrt{M_{1}}+1\right) M_{2}}{4} .
$$

This shows that the interference created by a signal belonging to one of $M_{2}$ constellation points increases the SER by a constant factor that depends on $M_{1}$ and $M_{2}$, instead of changing the dependence of the SER from the SNR to the SINR. Note that the above technique can be extended to the case with more than two users, where the SER bound would be increased by a factor of $\prod_{u=3}^{U} M_{u}$. As in most realistic environments there is only a limited number of dominant interferers, the SER bound remains manageable.

\section{SER Bound for the MAC Channel}

The preceding technique can in fact be applied to the case where the detector wishes to estimate not only the signal of user 1 , but also the signals of the other users. In this case,

$$
\operatorname{Pr}\left\{\left[\begin{array}{ll}
\hat{x}_{1} & \hat{x}_{2}
\end{array}\right]^{T} \neq\left[x_{1} x_{2}\right]^{T}\right\}=\operatorname{Pr}\left\{\hat{x}_{1} \neq x_{1}\right\}+\operatorname{Pr}\left\{\hat{x}_{2} \neq x_{2} \mid \hat{x}_{1}=x_{1}\right\} .
$$


The first term is the SER of user 1 in the presence of interference. The second term is the SER of user 2 in the absence of interference. Thus, when both users employ 4-QAM,

$$
p_{e, 12, \mathrm{JMD}, \mathrm{UB}}=p_{e, 1, \mathrm{JMD}, \mathrm{UB}}+q(\mathrm{INR}) \approx g(\mathrm{SIR}) \cdot \frac{1}{\mathrm{SNR}}=g\left(\frac{1}{\mathrm{SIR}}\right) \cdot \frac{1}{\mathrm{INR}},
$$

where

$$
g(\alpha)=1+\frac{\alpha}{2 \alpha+1}+\frac{9}{4} \cdot \frac{\alpha}{\alpha+1}+\frac{\alpha}{\alpha+2}+\alpha=f(\alpha)+\alpha
$$

The above approximation holds for high SNR or high INR. The coefficient $g(\alpha)$ is a monotonically increasing function of $\alpha$ for $0 \leq \alpha \leq 1$, and it is bounded as follows:

$$
g(0)=1 \leq g(\alpha) \leq \frac{91}{24}=g(1) .
$$

(49) clearly shows that for SIR $<1$ the SER is limited by SNR, whereas for SIR $\geq 1$ the SER is limited by INR. Thus, the SER is limited by the weaker signal, which agrees with intuition.

In general, when $x_{1}$ and $x_{2}$ belong to an $M_{1}$-QAM and an $M_{2}$-QAM constellation, respectively, an upper bound can be similarly derived. In fact, the SER can be bounded by the minimum of two ways of partitioning the error probability $\operatorname{Pr}\left\{\left[\begin{array}{ll}\hat{x}_{1} & \hat{x}_{2}\end{array}\right]^{T} \neq\left[\begin{array}{ll}x_{1} & x_{2}\end{array}\right]^{T}\right\}$, i.e. from (26) and (44), we have

$$
\begin{aligned}
& p_{e, 12, \mathrm{JMD}, \mathrm{UB}}= \\
& \quad \min \left\{\frac{\left(M_{1}-1\right)^{2} M_{2}}{6 \mathrm{SNR}}+2\left(1-\frac{1}{\sqrt{M_{2}}}\right) \frac{M_{2}-1}{3 \mathrm{INR}}, \quad \frac{\left(M_{2}-1\right)^{2} M_{1}}{6 \mathrm{INR}}+2\left(1-\frac{1}{\sqrt{M_{1}}}\right) \frac{M_{1}-1}{3 \mathrm{SNR}}\right\} .
\end{aligned}
$$

\section{ReCEIVER PERformance With TRANSmit DiVERsity}

This section shows that the use of the joint MD detector retains full diversity order in the presence of interference when simple transmit diversity schemes are employed. When the user of interest employs repetition coding over two time slots, or when both transmitters are synchronized and employ repetition coding over the same two time slots, a joint MD detector at receiver 1 achieves an SER proportional to $1 / \mathrm{SNR}^{2}$. This is in contrast to conventional detectors, where the limiting factor is $1 / \mathrm{SINR}^{2}$.

Consider the channel model (1) over two transmissions. In matrix form, we have

$$
\mathbf{y}^{T}=\overline{\mathbf{h}}^{*} \mathbf{X}+\mathbf{z}^{T}
$$

where $\mathbf{y}^{T}=[y[1] y[2]], \mathbf{z}^{T}=[z[1] z[2]], \overline{\mathbf{h}}^{*}=\left[h_{1}[1] h_{2}[1] h_{1}[2] h_{2}[2]\right]$, and $\mathbf{X}=\left[\begin{array}{cc}\mathbf{x}[1] & 0 \\ 0 & \mathbf{x}[2]\end{array}\right]$, where $\mathbf{x}[1]=\left[x_{1}[1] x_{2}[1]\right]^{T}, \mathbf{x}[2]=\left[x_{1}[2] x_{2}[2]\right]^{T}$. Similar to the analysis of Section VI, using transmit matrices 
$\mathbf{X}_{A}$ and $\mathbf{X}_{B}$ in place of transmit vectors, the average PEP between $\mathbf{X}_{A}$ and $\mathbf{X}_{B}$ is given by

$$
\operatorname{Pr}\left\{\mathbf{X}_{A} \rightarrow \mathbf{X}_{B}\right\}=\mathbb{E}_{\overline{\mathbf{h}}}\left[Q\left(\sqrt{\frac{\overline{\mathbf{h}}^{*}\left(\mathbf{X}_{A}-\mathbf{X}_{B}\right)\left(\mathbf{X}_{A}-\mathbf{X}_{B}\right)^{*} \overline{\mathbf{h}}}{2 N_{0}}}\right)\right]=\mathbb{E}_{\tilde{h}_{1}, \tilde{h}_{2}}\left[Q\left(\sqrt{\frac{\left|\tilde{h}_{1}\right|^{2} \bar{\lambda}_{1}^{2}+\left|\tilde{h}_{2}\right|^{2} \bar{\lambda}_{2}^{2}}{2 N_{0}}}\right)\right]
$$

where the matrix $\left(\mathbf{X}_{A}-\mathbf{X}_{B}\right)\left(\mathbf{X}_{A}-\mathbf{X}_{B}\right)^{*}$ is Hermitian and of rank 2, so its two nonzero eigenvalues are $\bar{\lambda}_{i}^{2}=\left\|\mathbf{X}_{A}(i)-\mathbf{X}_{B}(i)\right\|^{2}$, with $\mathbf{X}(i)$ denoting the $i$-th column of $\mathbf{X}$, and further we write $\left(\mathbf{X}_{A}-\right.$ $\left.\mathbf{X}_{B}\right)\left(\mathbf{X}_{A}-\mathbf{X}_{B}\right)^{*}=\overline{\mathbf{U}} \bar{\Lambda} \overline{\mathbf{U}}^{*}$ and define $\overline{\tilde{\mathbf{h}}} \triangleq \overline{\mathbf{U}}^{*} \overline{\mathbf{h}}=\left[\begin{array}{llll}\tilde{h}_{1} & \tilde{h}_{2} & \tilde{h}_{3} & \tilde{h}_{4}\end{array}\right]$. Substituting the expressions for the eigenvalues in (54), and using the fact that $Q(x) \leq e^{-x^{2} / 2}$ for $x>0$, and that, when $X$ is an exponential random variable, $\mathbb{E}\left[e^{s X}\right]=1 /(1-s)$ for $s<1[21]$, we have

$$
\begin{aligned}
\operatorname{Pr}\left\{\mathbf{X}_{A} \rightarrow \mathbf{X}_{B}\right\} & \leq \mathbb{E}_{\tilde{h}_{1}}\left[\exp \left(-\left|\tilde{h}_{1}\right|^{2} \frac{\left\|\mathbf{X}_{A}(1)-\mathbf{X}_{B}(1)\right\|^{2}}{4 N_{0}}\right)\right] \mathbb{E}_{\tilde{h}_{2}}\left[\exp \left(-\left|\tilde{h}_{2}\right|^{2} \frac{\left\|\mathbf{X}_{A}(2)-\mathbf{X}_{B}(2)\right\|^{2}}{4 N_{0}}\right)\right] \\
& =\left(\frac{4 N_{0}}{4 N_{0}+\left\|\mathbf{X}_{A}(1)-\mathbf{X}_{B}(1)\right\|^{2}}\right)\left(\frac{4 N_{0}}{4 N_{0}+\left\|\mathbf{X}_{A}(2)-\mathbf{X}_{B}(2)\right\|^{2}}\right)
\end{aligned}
$$

When a repetition code is employed by user 1 , the elements $x_{1}[1]$ and $x_{1}[2]$ of $\mathbf{X}$ are the same (but, not, necessarily $x_{2}[1]$ and $\left.x_{2}[2]\right)$. Assuming that 4-QAM is employed by both users, the average PEP does not depend on the transmitted matrix $\mathbf{X}$ because of the symmetry of 4-QAM. Thus, the transmit matrix $\mathbf{X}$ can be chosen to be $\mathbf{X}_{\mathbf{0}} \triangleq\left[\begin{array}{cc}\mathbf{x}_{0000} & 0 \\ 0 & \mathbf{x}_{0000}\end{array}\right]$ where $\mathbf{x}_{0000}=\left[\sqrt{\frac{P_{1}}{2}}(1+j) \sqrt{\frac{P_{2}}{2}}(1+j)\right]^{T}$. An upper bound for the SER can then be derived in a similar way to the analysis of Section VI by considering all transitions that lead to an incorrect decision for $x_{1}$. Using (55), after some algebra, it can be shown that

$$
p_{e 1, \mathrm{user}} 1 \text { rep.,JMD,UB }=\frac{k(\mathrm{SIR})}{\mathrm{SNR}^{2}},
$$

where

$$
\begin{aligned}
k(\alpha) & =9+\frac{35 \alpha}{1+\alpha}+\frac{16 \alpha}{2+\alpha}+\frac{8 \alpha}{1+2 \alpha}+\frac{32 \alpha^{2}}{(1+\alpha)^{2}}+\frac{16 \alpha^{2}}{(2+\alpha)^{2}} \\
& +\frac{32 \alpha^{2}}{(1+\alpha)(2+\alpha)}+\frac{8 \alpha^{2}}{(1+2 \alpha)(1+\alpha)}+\frac{8 \alpha^{2}}{(2+\alpha)^{2}} .
\end{aligned}
$$

Again, $k(\alpha)$ is a monotonically increasing function of $\alpha$ and can be bounded as

$$
9=k(0) \leq k(\alpha)<k(\infty)=156
$$

This bound in this case can be loose. However, it is interesting to note that nevertheless the diversity order is equal to 2 even when the second user does not use a diversity scheme, as long as the joint MD detector is employed by the user of interest. 
If a repetition code is employed by both users, and they are synchronized, $\bar{\lambda}_{1}=\bar{\lambda}_{2}=\left\|\mathbf{x}_{A}-\mathbf{x}_{B}\right\|$. Therefore (54) becomes

$$
\operatorname{Pr}\left\{\mathbf{x}_{A} \rightarrow \mathbf{x}_{B}\right\}=\mathbb{E}_{\tilde{h}_{1}, \tilde{h}_{2}}\left[Q\left(\sqrt{\frac{\left(\left|\tilde{h}_{1}\right|^{2}+\left|\tilde{h}_{2}\right|^{2}\right) \lambda_{1}^{2}}{2 N_{0}}}\right)\right] .
$$

The random variable $\|\tilde{h}\|^{2}=\left|\tilde{h}_{1}\right|^{2}+\left|\tilde{h}_{2}\right|^{2}$ follows a Chi-squared distribution with 4 degrees of freedom. It can be shown that, at high SNR [21],

$$
\operatorname{Pr}\left\{\mathbf{x}_{A} \rightarrow \mathbf{x}_{B}\right\}=\mathbb{E}_{\|\tilde{h}\|^{2}}\left[Q\left(\sqrt{\frac{\|\tilde{h}\|^{2} \bar{\lambda}_{1}^{2}}{2 N_{0}}}\right)\right] \approx\left(\begin{array}{c}
3 \\
2
\end{array}\right) \frac{1}{\left(\bar{\lambda}_{1}^{2} / N_{0}\right)^{2}}=\frac{3}{\left(\left\|\mathbf{x}_{A}-\mathbf{x}_{B}\right\|^{2} / N_{0}\right)^{2}} .
$$

By the union bound of probabilities,

$$
p_{e 1, \text { rep.,JMD,UB }} \approx \frac{27}{16} \frac{1}{\mathrm{SNR}^{2}}+\frac{51}{16} \frac{1}{(\mathrm{SNR}+\mathrm{INR})^{2}}+\frac{3}{2} \frac{1}{(\mathrm{SNR}+2 \mathrm{INR})^{2}}+\frac{3}{2} \frac{1}{(2 \mathrm{SNR}+\mathrm{INR})^{2}} .
$$

Thus, for sufficiently high SNR, the probability of error can be bounded by

$$
\operatorname{Pr}\left\{\hat{x}_{1} \neq x_{1}\right\} \lesssim l(\mathrm{SIR}) \cdot \frac{1}{\mathrm{SNR}^{2}}
$$

where

$$
l(\alpha)=\frac{27}{16}+\frac{51}{16} \frac{\alpha^{2}}{(\alpha+1)^{2}}+\frac{3}{2} \frac{\alpha^{2}}{(\alpha+2)^{2}}+\frac{3}{2} \frac{\alpha^{2}}{(2 \alpha+1)^{2}} .
$$

Note that the bound of (62) is approximate. Similar to $k(\alpha), l(\alpha)$ is a monotonically increasing function of $(\alpha)$ and can be bounded as follows:

$$
\frac{27}{16}=l(0) \leq l(\alpha)<l(\infty)=\frac{27}{4}
$$

Thus, when a simple repetition scheme is employed by both the user of interest and the interfering user, the joint MD detector achieves diversity of order 2. Moreover, the bound on the power loss is much smaller compared to the case when the interfering user does not employ a transmit diversity scheme.

In general, when user 1 employs $M_{1}$-QAM and user 2 employs a constellation of $M_{2}$ points, the following upper bounds can be derived, along the lines of Section VI-C.

$$
p_{e 1, \text { user } 1 \text { rep.,JMD,UB }} \approx\left(M_{1}-1\right) M_{2}^{2}\left(\frac{4 N_{0}}{4 N_{0}+\frac{6 P_{1}}{M_{1}-1}}\right)^{2}=\frac{4\left(M_{1}-1\right)^{3} M_{2}^{2}}{\left(2\left(M_{1}-1\right)+3 \mathrm{SNR}\right)^{2}}<\frac{4\left(M_{1}-1\right)^{3} M_{2}^{2}}{9 \mathrm{SNR}^{2}}
$$

when only the user of interest employs a repetition scheme, and

$$
p_{e 1, \text { rep. }, \mathrm{JMD}, \mathrm{UB}} \approx\left(M_{1}-1\right) M_{2} \frac{3}{\left(\frac{6 P_{1}}{N_{0}\left(M_{1}-1\right)}\right)^{2}}=\frac{\left(M_{1}-1\right)^{3} M_{2}}{12 \mathrm{SNR}^{2}}
$$


for the repetition scheme that involves both the user of interest and the interferer. Finally, we note that the above analysis can be easily extended to the case with repetition code over $d$ time slots and with advanced diversity coding schemes. Table II summarizes the main results of Sections VI and VII.

\section{Simulation Results}

In this section, the performance of various detectors is evaluated by Monte Carlo simulation to verify the theoretical results. Results for the AWGN case are shown first. Fig. 1 shows the SER of the optimal ML (and also joint MD) detector when 2-PAM is used by both transmitters in a two-user IC for various SIR levels. As expected, the detector performs best when SIR is infinite, i.e., when there is no interference. As the interference power increases for the fixed desired signal power, the performance of the detector degrades until $\mathrm{SIR}=1$. As the interference power increases beyond the signal power, the performance actually improves. Thus, optimal ML (also joint MD) detection implicitly subtracts the interference when SIR is small. Note that the detection is not subject to an error floor, regardless of the value of SIR.

Fig. 2 shows the SER performance when 4-PAM is used at both transmitters. Both a low-interference case $(\mathrm{SIR}=3 \mathrm{~dB})$ and a high-interference case $(\mathrm{SIR}=-3 \mathrm{~dB})$ are considered. In both cases, the MD and ML detectors perform well, while the interference-ignorant and the SIC detectors exhibit error floors. This agrees with Proposition 1, as $\mathrm{SIR}_{t h, I I}=9.54 \mathrm{~dB}, \mathrm{SIR}_{t h, S I C, 1}=-9.54 \mathrm{~dB}$ and $\mathrm{SIR}_{t h, S I C, 2}=15.56 \mathrm{~dB}$ for 4-PAM, and $\mathrm{SIR}= \pm 3 \mathrm{~dB}$ are in the range where both conventional detectors fail to perform satisfactorily. Thus, the use of the joint MD or ML detectors is crucial for enabling 4-PAM signaling in this SIR range over the IC, which would otherwise be impossible. A similar phenomenon occurs for the 3-user IC as shown in Fig. 3, where the SER with all three users employing 2-PAM is plotted. As can be seen, the joint $\mathrm{MD}$ and the optimal ML detectors perform well for $\mathrm{SIR}=1 \mathrm{~dB}$ and $-1 \mathrm{~dB}$, while both the interference-ignorant and the SIC detectors exhibit an error floor.

For the performance of various detectors in a fading environment, Fig. 4 shows the SER when both users employ 4-QAM and SIR =12dB. As can be seen, in the absence of the interference, the SER curve follows the theoretical 1/SNR curve quite well. The presence of interference increases the SER. However, when the joint MD or the optimal ML detector are used, the SER curve continues to behave as $c /$ SNR. Theoretical calculation in Section VI-C suggests $c=f(\operatorname{SIR})=4.49$, which agrees with the numerical simulation as shown in the figure. Note that the interference-ignorant detector, the SIC detector, and the ordered SIC detector all have error floors. The figure also shows that when the joint MD detector tries to 
detect both users' signals, the SER is approximated by the theoretical curve $g(\mathbf{S I R}) / \mathrm{SNR}=20 / \mathrm{SNR}$.

Figs. 5 and 6 show the performance of various detectors when both users employ QAM with $\left(M_{1}, M_{2}\right)=$ $(16,4)$ and $(16,16)$. In both figures, the simulation results again agree reasonably well with the theoretical approximate upper bound (46). In the figure, the ordered SIC is based on the instantaneous minimum distance of the received constellations of user 1 and 2. As can be seen, both the interference-ignorant and SIC detectors show error floors. Moreover, Fig. 6 shows that the interference-ignorant detector is limited by SINR for 16-QAM even though the exact analysis was done only for PSK in Section VI-B.

Fig. 7 considers a 3 -user IC with all three users employing 4-QAM, and SIR $=\frac{P_{1}}{P_{2}+P_{3}}=12 \mathrm{~dB}$. Simulation shows that the SER of the joint MD detector follows the theoretical upper bound very well, which is $M_{3} f(2 \mathrm{SIR}) / \mathrm{SNR}=4 f\left(2 \times 10^{1.2}\right) / \mathrm{SNR} \approx 18.46 / \mathrm{SNR}$ since $P_{1} / P_{2}=2 \mathrm{SIR}$, whereas the interference-ignorant detector shows an error floor in the presence of two interferers.

Fig. 8 shows the performance of various detectors when repetition coding is used by both users. Here the modulation scheme is 4-QAM for both users. As can be seen in the figures, with repetition coding, the diversity order becomes 2 when the joint MD detector is used. On the other hand, the SER for the interference-ignorant detector is approximated by $\frac{3}{2 \operatorname{SINR}^{2}}$, which is interference limited.

\section{CONCLUSiON}

This paper analyzed the performance of the joint MD and the optimal ML detectors for the Gaussian IC where discrete constellations are used by the transmitters. Unlike the interference-ignorant detector, these joint detectors exploit knowledge of the distribution of the interference rather than treating the interference as Gaussian noise. Furthermore, unlike the SIC detector, no explicit decision on the interfering signal is made by the detectors, thereby avoiding error propagation. The SER performance of these detectors was compared analytically and by simulation. In AWGN channels, the joint MD and the optimal ML detectors perform well in the presence of interference for almost all SIR values, whereas the performance of the interference-ignorant and the SIC detectors suffers from error floors for some SIR values. These results suggest that an optimal detector for the Gaussian IC should neither completely ignore nor completely decode the interference-it should do something in-between.

The absence of error floors for the optimal ML and the joint MD detectors makes their use attractive when the channel varies constantly. This paper shows that their use in fading channels significantly improves the SER performance as compared to conventional detectors in the sense that the SER be- 
comes inversely proportional to the SNR rather than the SINR. In other words, joint detection turns an interference-limited channel into a noise-limited channel. This is particularly desirable in distributed BWC systems where sophisticated joint receivers can be used to improve spectral efficiency. In such systems joint detection can even be performed at a central unit by relying on the backbone that conveys the signals received at the distributed locations.

Moreover, it was shown that when advanced joint detectors are employed, full diversity gains can be achieved even in the presence of interference. This implies that the use of transmit diversity schemes is advantageous as compared to orthogonalization schemes that avoid interference systematically and offers a distributed BWC the flexibility to provide multiple simultaneous connections with full diversity gains. Therefore, although advanced detection schemes require more information about interferers and are more complex to implement, the improved performance can make their use attractive in future distributed BWC systems.

\section{ACKNOWLEDGMENT}

Work of the second author has been performed in the framework of the FUTON-FP7-ICT-2007-215533 project, which is partially funded by the European Commission. The third author wishes to acknowledge the support of the Natural Science and Engineering Research Council (NSERC) of Canada.

\section{REFERENCES}

[1] http://www.itu.int/md/R07-IMT.ADV-C.

[2] http://www.3gpp.org/LTE-Advanced.

[3] http://wirelessman.org/tgm/.

[4] X. You and G. Chen, "Towards beyond 3G - the FuTURE project in China," China Communications, Dec. 2004.

[5] "Fibre-optic networks for distributed extendible heterogeneous radio architectures and service provisioning (ICT-2007-215533FUTON)," http://www.ict-futon.eu.

[6] W. Yu, G. Ginis, and J. M. Cioffi, "Distributed multiuser power control for digital subscriber lines," IEEE J. Select. Areas Commun., vol. 20, pp. 1105-1115, June 2002.

[7] R. Cendrillon, W. Yu, M. Moonen, J. Verlinder, and T. Bostoen, "Optimal multi-user spectrum management for digital subscriber lines," IEEE Trans. Commun., vol. 54, pp. 922-933, May 2006.

[8] J. Lee, R. V. Sonalkar, and J. M. Cioffi, "Multi-user discrete bit-loading for DMT-based DSL systems," in IEEE Global Telecommun. Conf. (GLOBECOM), vol. 2, 2002, pp. 1259-1263.

[9] J. Wang and L. B. Milstein, "CDMA overlay situations for microcellular mobile communications," IEEE Trans. Commun., vol. 43, pp. 603-614, Feb./Mar./Apr. 1995. 
[10] J. Wang and J. Chen, "Performance of wideband CDMA systems with complex spreading and imperfect channel estimation," IEEE J. Select. Areas in Commun., vol. 19, pp. 152-163, Jan. 2001.

[11] S. Verdú, Multiuser Detection. Cambridge Univ. Press, 1998.

[12] Z. Zvonar and D. Brady, "Multiuser detection in single-path fading channels," IEEE Trans. Commun., vol. 42, no. 2/3/4, pp. 1729-1739, 1994.

[13] S. J. Grant and J. K. Cavers, "Further analytical results on the joint detection of cochannel signals using diversity arrays," IEEE Trans. Commun., vol. 48, no. 11, pp. 1788-1791, Nov. 2000.

[14] J. Kazemitabar and H. Jafarkhani, "Performance analysis of multiple antenna multi-user detection," in Information Theory and Applications Workshop, 2009.

[15] G. Caire, G. Taricco, J. Ventura-Traveset, and E. Biglieri, “A multiuser approach to narrowband cellular communications,” IEEE Trans. Inf. Theory, vol. 43, no. 5, pp. 1503-1517, Sep. 1997.

[16] S. J. Grant and J. K. Cavers, "System-wide capacity increase for narrowband cellular systems through multiuser detection and base station diversity arrays," IEEE Trans. Wireless Commun., vol. 3, no. 6, pp. 2072-2082, Nov. 2004.

[17] H. Dai and H. V. Poor, "Asymptotic spectral efficiency of multicell MIMO systems with frequency-flat fading," IEEE Trans. Signal Process., vol. 51, no. 11, pp. 2976-2988, Nov. 2003.

[18] R. H. Etkin, D. Tse, and H. Wang, "Gaussian interference channel capacity to within one bit," IEEE Trans. Inf. Theory, vol. 54, no. 12, pp. 5534-5562, Dec. 2008

[19] J. M. Cioffi, "Course notes for digital communication: Signal processing," http://www.stanford.edu/group/cioffi/.

[20] J. Chen, J. Wang, and M. Sawahashi, "MCI cancellation for multicode wideband CDMA systems," IEEE J. Select. Areas in Commun., vol. 20, pp. 450-462, Feb. 2002.

[21] D. Tse and P. Viswanath, Fundamentals of Wireless Communications. Cambridge Univ. Press, 2005. 
TABLE I

SIR THRESHOLD VALUES FOR THE INTERFERENCE-IGNORANT DETECTOR AND THE SIC DETECTOR

\begin{tabular}{|c|c|c|c|}
\hline$\left(M_{1}, M_{2}\right)$ & $\operatorname{SIR}_{t h, I I}(\mathrm{~dB})$ & $\operatorname{SIR}_{t h, S I C, 1}(\mathrm{~dB})$ & $\operatorname{SIR}_{t h, S I C, 2}(\mathrm{~dB})$ \\
\hline$(2,2)$ & 0 & 0 & 6.02 \\
\hline$(2,4)$ & 2.55 & -6.99 & 8.57 \\
\hline$(2,8)$ & 3.68 & -13.22 & 9.70 \\
\hline$(4,2)$ & 6.99 & -2.55 & 13.01 \\
\hline$(4,4)$ & 9.54 & -9.54 & 15.56 \\
\hline$(4,8)$ & 10.67 & -15.77 & 16.69 \\
\hline$(8,2)$ & 13.22 & -3.68 & 19.24 \\
\hline$(8,4)$ & 15.77 & -10.67 & 21.80 \\
\hline$(8,8)$ & 16.90 & -16.90 & 22.92 \\
\hline
\end{tabular}

TABLE II

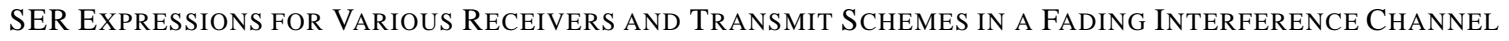

\begin{tabular}{|c|c|}
\hline Detector & Probability of Error \\
\hline Interference-Ignorant Detector & $P\left\{\hat{x}_{1} \neq x_{1}\right\} \lesssim 2\left(1-\frac{1}{\sqrt{M_{1}}}\right) \frac{M_{1}-1}{3 \operatorname{SINR}}$ \\
\hline Joint MD for the desired signal & $P\left\{\hat{x}_{1} \neq x_{1}\right\} \lesssim \frac{\left(M_{1}-1\right)^{2} M_{2}}{6 \mathrm{SNR}}$ \\
\hline Joint MD for the desired signal and the interfering signal & 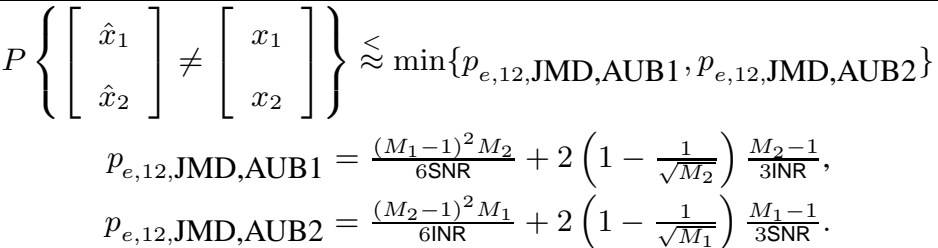 \\
\hline Repetition coding (user 1), Joint MD for the desired signal & $P\left\{\hat{x}_{1} \neq x_{1}\right\} \lesssim \frac{4\left(M_{1}-1\right)^{3} M_{2}^{2}}{9 \mathrm{SNR}^{2}}$ \\
\hline Repetition coding (users 1 and 2), Joint MD for the desired signal & $P\left\{\hat{x}_{1} \neq x_{1}\right\} \lesssim \frac{\left(M_{1}-1\right)^{3} M_{2}}{12 \mathrm{SNR}^{2}}$. \\
\hline
\end{tabular}

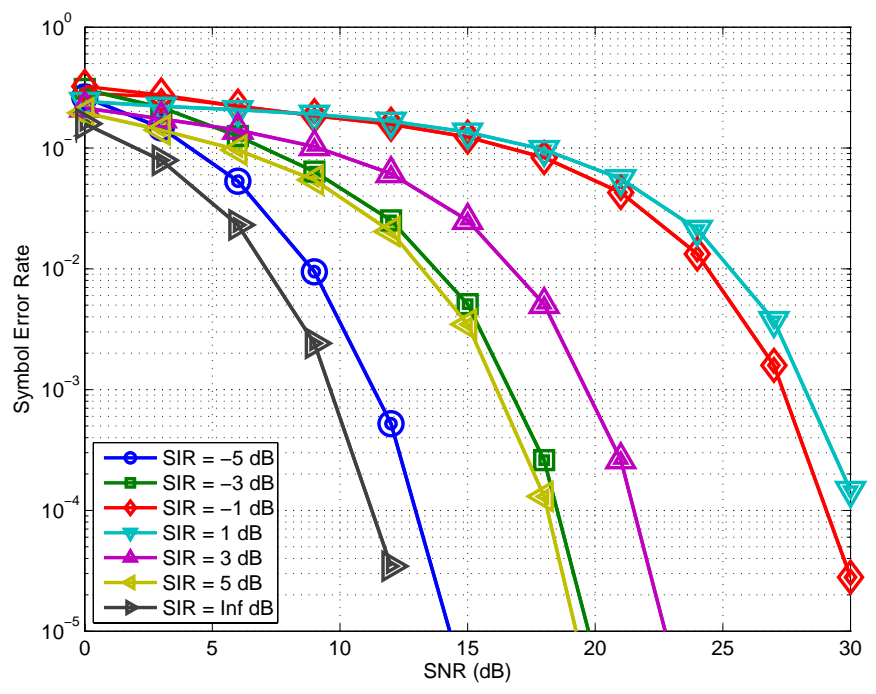

Fig. 1. SER for the optimal ML and the joint MD detector in AWGN channels for various SIR values when 2-PAM is used by both transmitters. Curves for the optimal ML detector and the joint MD detector overlap. 


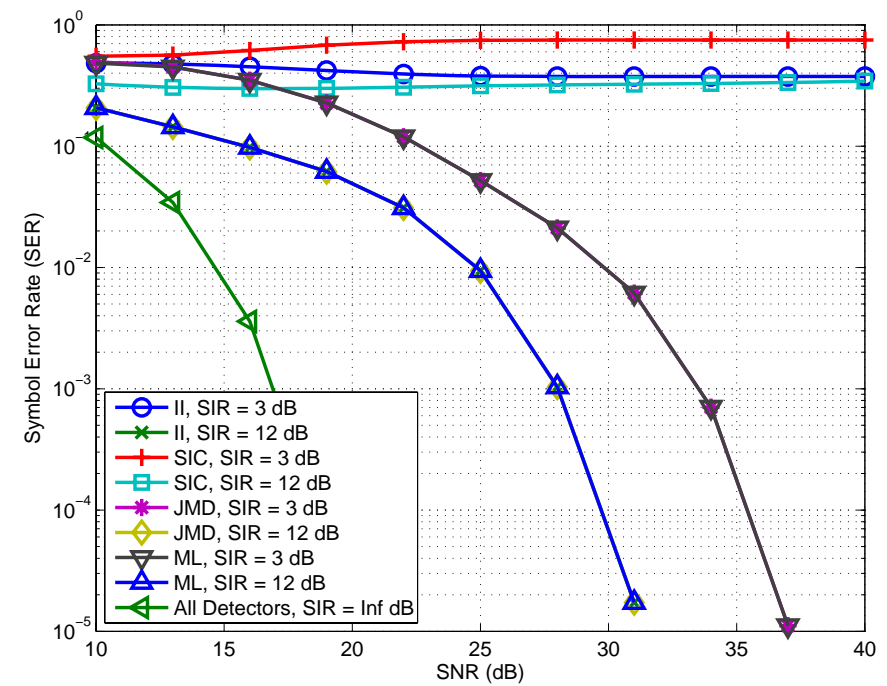

Fig. 2. SER for all 4 detectors in AWGN channels when 4-PAM is used by both transmitters.

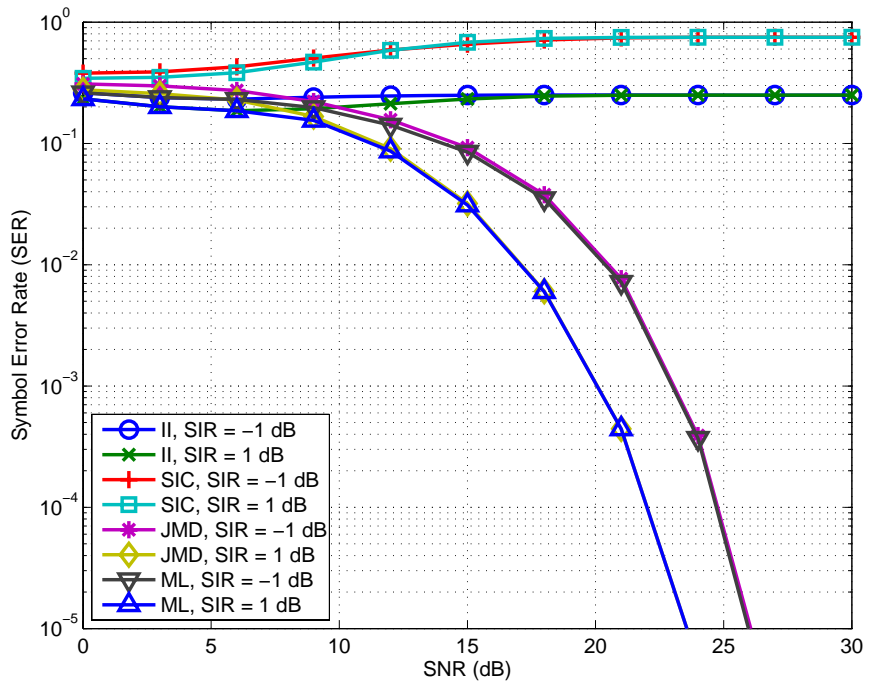

Fig. 3. SER for all 4 detectors in a 3-user AWGN IC when 2-PAM is used by all users. It is assumed that each interference has the same power. 


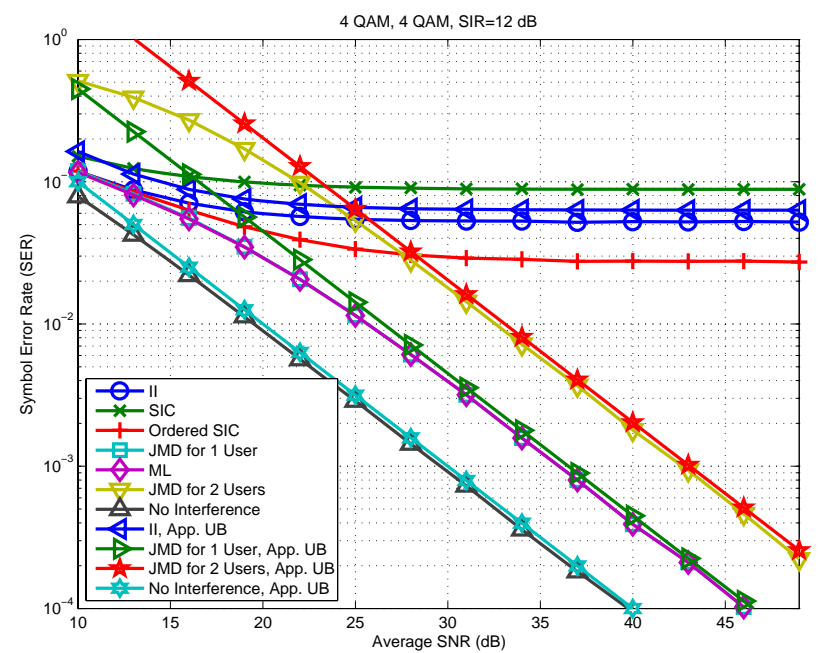

Fig. 4. SER of various detectors in Rayleigh fading channels with 2 users employing 4-QAM and 4-QAM for SIR $=12$ dB.

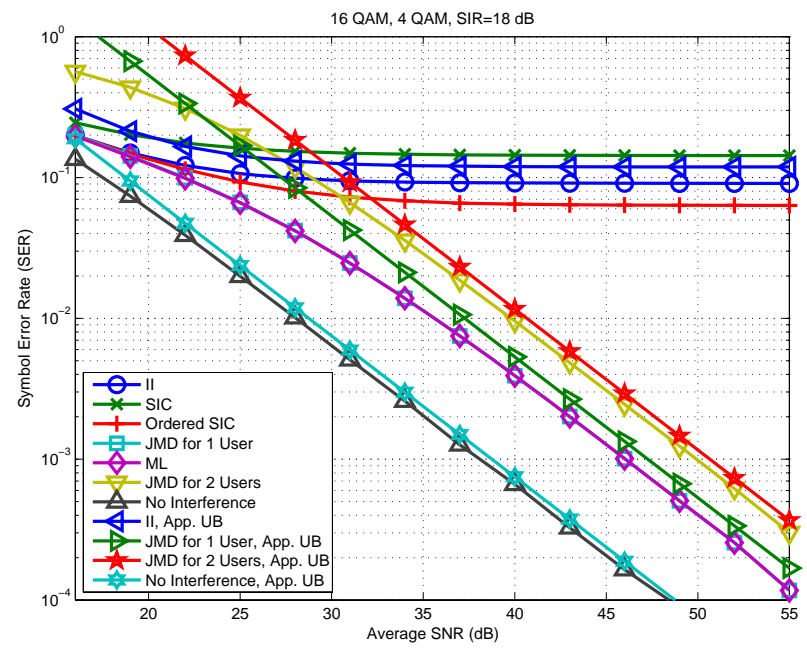

Fig. 5. SER of various detectors in Rayleigh fading channels with 2 users employing 16-QAM and 4-QAM for SIR $=18 \mathrm{~dB}$.

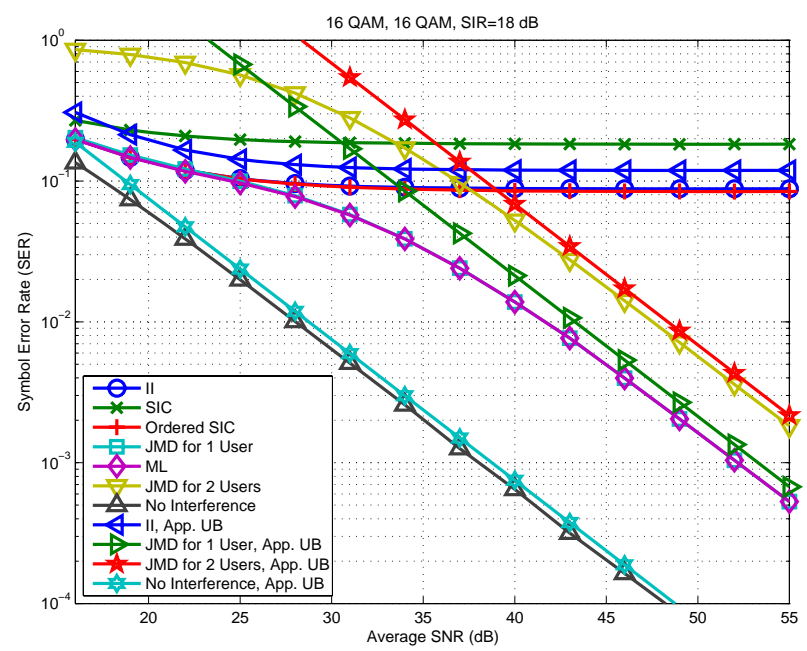

Fig. 6. SER of various detectors in Rayleigh fading channels with 2 users employing 16-QAM and 16-QAM for SIR $=18 \mathrm{~dB}$. 


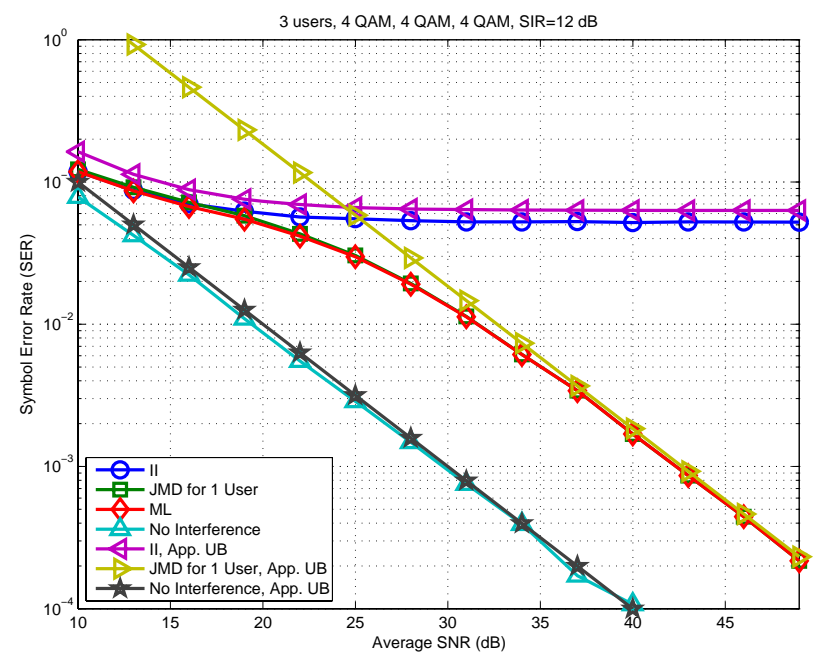

Fig. 7. SER of various detectors in Rayleigh fading channels with three users all employing 4-QAM for SIR $=12 \mathrm{~dB}$.

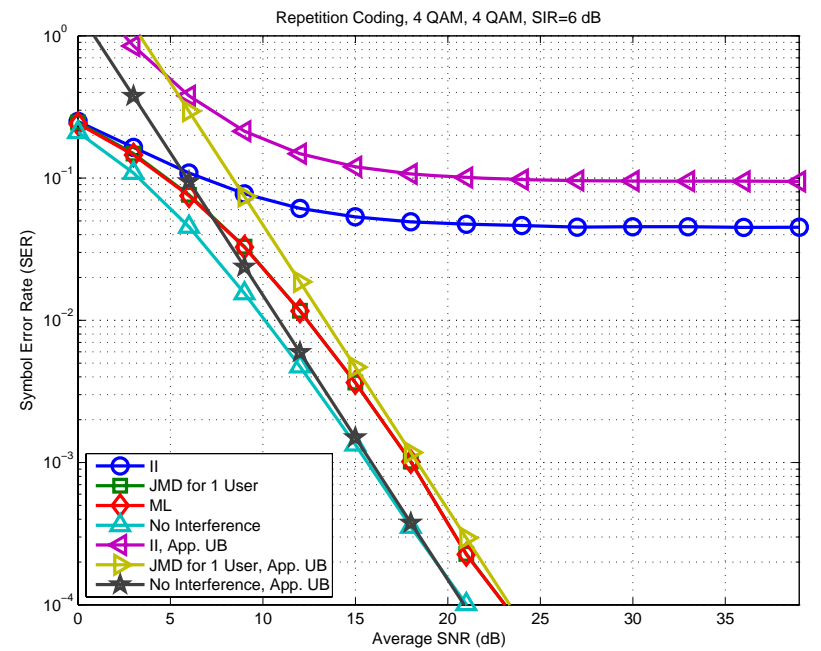

Fig. 8. SER with repetition coding with both users employing repetition coding with 4-QAM for SIR $=6 \mathrm{~dB}$. 